\title{
Reversibility of stress-echo induced ST-segment depression by long-term oral n-3 PUFA supplementation in subjects with chest pain syndrome, normal wall motion at stress-echo and normal coronary angiogram
}

\author{
Nicola Gaibazzi* and Vigilio Ziacchi
}

Address: Cardiology Department, Ospedale Civile di Desenzano del Garda, Desenzano del Garda (BS), Italy

Email: Nicola Gaibazzi* - nicola.gaibazzi@inwind.it; Vigilio Ziacchi - vigilio.ziacchi@aod.it

* Corresponding author

Published: 23 March 2004

BMC Cardiovascular Disorders 2004, 4:1

This article is available from: http://www.biomedcentral.com/|47|-226|/4/I

(c) 2004 Gaibazzi and Ziacchi; licensee BioMed Central Ltd. This is an Open Access article: verbatim copying and redistribution of this article are permitted in all media for any purpose, provided this notice is preserved along with the article's original URL.
Received: 07 November 2003

Accepted: 23 March 2004

\begin{abstract}
Background: Normal coronary arteries may coexist with abnormal coronary and systemic endothelial function in patients with chest pain. Recent work by the renowned Pisa echo-group elegantly suggests that isolated ST-segment depression during stress-echo (SE) can be used as a marker of coronary endothelial dysfunction, in the absence of stress-inducible wall motion abnormalities and in the absence of angiographically-significant coronary artery disease (CAD). The long chain n-3 polyunsaturated fatty acids (PUFAs) have been reported to possess several properties that may positively influence vascular function. The present study's hypothesis is that a 4 month-course of oral supplementation with n-3 PUFAs can reverse endothelial dysfunction.
\end{abstract}

Methods: Subjects were selected on the basis of the following criteria: I) reported chest pain syndrome, 2) significant ST-segment depression during an otherwise normal SE, 3) absence of angiographically-significant CAD. Subjects underwent a 4-month course of oral supplementation with commercially available n-3 PUFA, I g once a day. Normalization of endothelial dysfunction was defined, at the end of the supplementation period, by the absence of significant ST-segment depression during repeat SE. We tested the aforementioned hypothesis in a very small series of consecutive subjects, with the intent to produce a hypothesis-generating study.

Results: Seven out of the total nine subjects enrolled (77.8\%) had normal ST-segment during repeat SE performed after the 4 month course of therapy.

Conclusions: A striking rate of reversion of SE-induced ST-segment depression after oral n-3 PUFAs suggests reversion of coronary endothelial dysfunction; nonetheless these data need to be validated in larger, placebo-controlled studies.

Background

Normal coronary arteries may coexist with abnormal cor- onary and systemic endothelial function in patients with chest pain [1]. The functional status of systemic endothe- 
lium somewhat mirrors the coronary endothelial function $[2,3]$ and can be evaluated non-invasively with brachial ultrasound [4]. A novel, provocative paper by the Pisa echo-lab group $[5,6]$, suggests that endothelial dysfunction strictly correlates with ST-segment depression (STSD) during pharmacologic (as well as exercise) stress, while epicardial coronary artery stenosis exclusively affects wall motion abnormalities (WMA), although both components may coexist; they demonstrated that abnormal brachial artery flow-mediated dilation (measured with brachial artery ultrasound) was predicted by STSD during SE but not by either presence of WMA during SE or angiographically significant CAD. There was no correlation between brachial artery flow-mediated dilation and extent of angiographical CAD.

Therefore isolated STSD during SE could be used as a surrogate for coronary endothelial dysfunction, in the absence of stress-inducible WMA and in the absence of angiographically-significant CAD.

The majority of cardiovascular benefits of PUFAs are likely to be mediated in the vascular wall and at the vascular endothelium level. The long chain PUFAs have been reported to possess several properties that may positively influence vascular function. These include favorable mediator profiles (nitric oxide, eicosanoids) that influence vascular reactivity, change in vascular tone via actions on selective ion channels [7], and maintenance of vascular integrity. In addition to direct effects on contractility, PUFAs may affect vascular function by modifying expression of inflammatory cytokines and adhesion molecules. These properties may explain the beneficial cardiovascular protection of this family of fatty acids that have been clearly evident through epidemiological data as well as from few clinical trials $[8,9]$.

Although many epidemiological surveys and basic research studies have suggested that "marine fish oil" cardioprotective effect is consistent and at least partly mediated through an action on the endothelium, no large clinical intervention trial has yet addressed the effect of commercially-available PUFAs preparations on a clinically-measurable direct marker of coronary endothelial dysfunction in normocholesterolemic subjects $[10,11]$. A small, randomized study performed in hypercholesterolemic subjects suggests a positive effect of 4-month marine oil supplementation on brachial artery endothelium-mediated vasodilation.[12]

The present study's hypothesis is that a 4 month-course of oral supplementation with PUFAs can reverse coronary endothelial dysfunction. Eventual "normalization" of endothelial dysfunction was measured, at the end of the supplementation period, by the absence of both significant STSD and WMA during repeat SE.

\section{Methods}

Patients were enrolled in the study if they met the following inclusion criteria: (1) chest pain syndrome; (2) reference for SE; (3) presence of STSD (strict ECG criteria were applied: a) STSD $\pm 1 \mathrm{~mm} 0.08 \mathrm{~s}$ after the J point in the peripheral leads and/or b) STSD $\geq 2 \mathrm{~mm} 0.08 \mathrm{~s}$ after the J point in the precordial leads) during a SE with no changes in wall motion score, (4) coronary angiogram performed within 20 days after SE, (5) no nitrate therapy at the time of SE.

When meeting the aforementioned entry criteria, patients had their total cholesterol measured by means of a standard whole-blood sample.

Subjects were referred for SE because of one of the following: (a) patients with positive exercise-ECG in concomitance with conditions lowering the positive predictive value of the ECG marker of ischaemia (for instance, female gender or arterial hypertension); (b) patients with recurring chest pain in the absence of significant electrocardiographic changes during routine exercise-ECG; (c) patients unable to exercise. All of the patients underwent dipyridamole SE.

The following were exclusion criteria: (1) uninterpretable resting ECG (left bundle branch block, preexcitation, baseline STSD or elevation $>1 \mathrm{~mm}$, or typical digitalis repolarization pattern); (2) technically poor acoustic window; (3) cardiomyopathy or severe valvular disease; (4) previously detected CAD; (5) statin therapy; (6) high total cholesterol levels (defined as total cholesterol $\geq 240 \mathrm{mg}$ / $\mathrm{dl}$ as suggested by current NCEP guidelines).

The study was approved by the institutional review board. All patients gave their written informed consent before entering the study.

Stress echo was performed with dipyridamole up to 0.84 $\mathrm{mg} / \mathrm{kg}$ over $10 \mathrm{~min}$ with atropine up to $2 \mathrm{mg}$ when needed; stress echo was repeated using the same protocol after a 4-month course of oral supplementation with commercially available $\left(\right.$ Seacor $^{\circledR}$, Esapent ${ }^{\circledast}$, Eskim $\left.^{\circledR}\right)$ n-3 PUFA at a dose of $1 \mathrm{~g}$ once a day. An imaging system with digital acquisition was used (GE-Vivid 7). All standard echocardiographic views were obtained when possible. The left ventricle was divided into 16 segments, as suggested by the American Society of Echocardiography. Segmental wall motion was graded as follows: normal $=1$; hypokinetic $=2$; akinetic $=3$; and dyskinetic $=4$. Inadequately visualized segments were not scored. Stress echo was considered positive when one left ventricular segment was 
increased by one grade or more at peak stress. Normalization of coronary endothelial dysfunction was defined as isoelectric ST segment $0.08 \mathrm{~s}$ after the $\mathrm{J}$ point in the peripheral leads and STSD $<0.5 \mathrm{~mm} 0.08 \mathrm{~s}$ after the J point in the precordial leads during repeat SE with no WMA.

Coronary angiography in multiple views was performed according to the standard Judkins or Sones technique.

The percent diameter stenosis was determined by quantitative coronary angiography. A vessel was considered to have significant obstruction if its diameter was narrowed by $50 \%$ or more, with respect to the pre-stenotic tract.

\section{Results}

Nine consecutive patients were prospectively enrolled according to inclusion/exclusion criteria during the time period between January 2003 and June 2003. Three patients (out of the 12 who fulfilled entry criteria) were excluded because of the presence of at least one exclusion criteria (2 subjects had high total cholesterol levels as per protocol defined and one was already on statin therapy).

Five patients were male (55\%); mean age was $60( \pm 5)$.

Four patients (44\%) had hypertension (treated with ACEinhibitors, ARBs, diuretics or combinations of them), $3(33 \%)$ had diabetes (treated with a combination of metformin and a sulfonylurea), 2(22\%) had total cholesterol levels in the range between $200 \mathrm{mg} / \mathrm{dl}$ and $240 \mathrm{mg} / \mathrm{dl}$ (defined as borderline high by current NCEP guidelines), $3(33 \%)$ were active smokers. None was under antiischaemic therapy (beta-blocker and/or non-dihydropiridinic calcium antagonist) at the time of SE testing. All of the patients maintained their usual therapy througout the study time period, with no exceptions.

All but 2 subjects had normalization of STSD during repeat SE performed after the 4 month course of therapy. Of the two subjects still presenting STSD at repeat SE, one was hypertensive and non-diabetic while the other was diabetic but non-hypertensive).

\section{Discussion}

We tested the study hypothesis in a small series of consecutive subjects, with the intent to produce a small hypothesis-generating study; testing the hypothesis in a large, randomized, placebo-controlled trial was felt premature, both because we used an innovative, but not definitely validated marker of coronary endothelial dysfunction and because lack of strong clinical data about the effect of PUFAs supplementation on human coronary endothelium.
Our study, as any small "proof of concept" study, has many limitations:

1) It is a prospective, non-randomized, uncontrolled interventional study, 2) it is performed in a very small group of subjects, 3 ) it is suggestive for a positive-reversal effect of PUFAs on isolated, angiographically-negative STSD during SE, but even if recent data strongly suggest this clinical pattern to be linked with coronary endothelial dysfunction $(5,6)$, this is not a direct measure of endothelial function and, also, no data exist demonstrating that reversal of STSD indicates normalization of coronary endothelial dysfunction.

Of course no subgroup analysis can be performed in such a small group of subjects, even if it is interesting that nonresponders (2 out of 9 subjects) consisted of one hypertensive, non-diabetic subject and one diabetic non-hypertensive subject, suggesting that inefficacy of therapy may not be linked to the presence/absence of these clinical conditions.

Even if evidence is strong for a negative prognostic effect of coronary endothelial dysfunction on mortality, atherosclerosis progression and plaque instability [13], coronary endothelial function is to date difficult to measure on clinical grounds: hence it is obvious cardiologists prefer to dilate what they can clearly see (epicardial stenosis) rather than pharmacologically engage what they can not measure (endothelium).

An easy to obtain, clinically useful marker of coronary endothelial dysfunction such as isolated STSD during SE in the absence of visible CAD would be very welcome in the cardiology arena, while the striking effect exerted by PUFAs in this small study would be even more valuable.

\section{Conclusions}

Striking rate of reversion of stress-induced ST-segment depression after oral n-3 PUFAs suggests reversion of coronary endothelial dysfunction; nonetheless these data need to be validated in larger, placebo-controlled studies utilizing direct measurements of coronary endothelial function.

\section{Competing interests}

None declared.

\section{Authors' contributions}

NG conceived the study and materially performed echocardiographic examinations, VG participated in the coordination of the study and revision of the paper. All authors read and approved the final manuscript. 


\section{References}

I. Egashira K, Inou T, Hirooka , Yamada A, Urabe Y, Takeshita A: Evidence of impaired endothelium-dependent coronary vasodilation in patients with angina pectoris and normal coronary angiograms. $N$ Engl J Med I993, 328: |659-64.

2. Anderson TJ, Uehata A, Gerhard MD, Meredith IT, Knab S, Delagrange D, Lieberman EH, Ganz P, Creager MA, Yeung AC: Close relationship of endothelial function in the human coronary and peripheral circulations. J Am Coll Cardiol 1995, 26: I235-4 I.

3. Takase B, Uehata A, Akima T, Nagai T, Nishioka T, Hamabe A, Satomura K, Ohsuzu F, Kurita A: Endothelium-dependent flowmediated vasodilation in coronary and brachial arteries in suspected coronary artery disease. Am / Cardiol I 998, 82: I535-9.

4. Celermajer DS, Sorensen KE, Gooch VM, Spiegelhalter DJ, Miller OI, Sullivan ID, Lloyd JK, Deanfield JE: Non-invasive detection of endothelial dysfunction in children and adults at risk of atherosclerosis. Lancet 1992, 340: IIII-I5.

5. Palinkas A, Toth E, Amyot R, Rigo F, Venneri L, Picano E: The value of ECG and echocardiography during stress testing for identifying systemic endothelial dysfunction and epicardial artery stenosis. Eur Heart J 2002, 23(20): I587-95.

6. Preda I: Differentiation between endothelial dysfunction and epicardial coronary artery stenosis with the aid of stress ECG and echocardiography. A novel return of the old ECG! Eur Heart J 2002, 23(20): | 56 |-2.

7. Ye D, Zhang D, Oltman C, Dellsperger K, Lee HC, VanRollins M: Cytochrome P-450 Epoxygenase Metabolites of Docosahexaenoate Potently Dilate Coronary Arterioles by Activating Large-Conductance Calcium-Activated Potassium Channels. J Pharmacol Exp Ther 2002, 303(2):768-76.

8. Abeywardena MY, Head RJ: Longchain $\mathbf{n}-3$ polyunsaturated fatty acids and blood vessel function. Cardiovasc Res 200I, 52(3):36I-7I.

9. Dietary supplementation with $n-3$ polyunsaturated fatty acids and vitamin $E$ after myocardial infarction: results of the GISSI-Prevenzione trial. Gruppo Italiano per lo Studio della Sopravvivenza nell'Infarto Miocardico. Lancet 1999, 354:447-55

10. Kromhout D, Bosschieter EB, de Lezenne Coulander C: The inverse relation between fish consumption and 20 year mortality from coronary heart disease. $N$ Engl J Med I 985, 3 I 2: I 205-9.

II. Goode GK, Garcia S, Heagerty AM: Dietary supplementation with marine fish oil improves in vitro small artery endothelial function in hypercholesterolemic patients: a double-blind placebo-controlled study. Circulation 1997, 96:2802-7.

12. Goodfellow J, Bellamy MF, Ramsey MW, Jones C], Lewis MJ: Dietary supplementation with marine omega-3 fatty acids improve systemic large artery endothelial function in subjects with hypercholesterolemia. I Am Coll Cardiol 2000, 35(2):265-70.

13. Halcox JP, Schenke WH, Zalos G, Mincemoyer R, Prasad A, Waclawiw MA, Nour KR, Quyyumi AA: Prognostic value of coronary vascular endothelial dysfunction. Circulation 2002, 106(6):653-8.

\section{Pre-publication history}

The pre-publication history for this paper can be accessed here:

http://www.biomedcentral.com/1471-2261/4/1/prepub
Publish with Biomed Central and every scientist can read your work free of charge

"BioMed Central will be the most significant development for disseminating the results of biomedical research in our lifetime. "

Sir Paul Nurse, Cancer Research UK

Your research papers will be:

- available free of charge to the entire biomedical community

- peer reviewed and published immediately upon acceptance

- cited in PubMed and archived on PubMed Central

- yours - you keep the copyright
BioMedcentral 